\title{
Erratum to: Dioscin-induced autophagy mitigates cell apoptosis through modulation of PI3K/Akt and ERK and JNK signaling pathways in human lung cancer cell lines
}

\author{
Ming-Ju Hsieh" ${ }^{1}$ Te-Lung Tsai ${ }^{2,3}$ • Yih-Shou Hsieh ${ }^{2,4}$ • Chau-Jong Wang ${ }^{2,5}$. \\ Hui-Ling Chiou ${ }^{1,6}$
}

Published online: 27 April 2017

(C) Springer-Verlag Berlin Heidelberg 2017

\section{Erratum to: Arch Toxicol (2013) 87:1927-1937 DOI 10.1007/s00204-013-1047-z}

The original publication of this article unfortunately contained errors in Figure 2D, 3A and Figure 5ABCD, which has been corrected in the figures below. In the original article, Figure 2D contained an error. The cleaved PARP panel in H1299 is wrong and appears as a duplicate of the cleaved PARP panel in A549. In Figure 3A, the wrong image in dioscin-treatment $\mathrm{H} 1299$ cells (Dioscin, $1.25 \mu \mathrm{M}$ ) appears as a duplicate of the image in dioscin-treatment A549 cells (Dioscin, $1.25 \mu \mathrm{M}$ ). In Figure 5A, the wrong p-mTOR panel in dioscin-treatment A549 appears as the other panel. In

The online version of the original article can be found under doi:10.1007/s00204-013-1047-z.

Chau-Jong Wang

wcj@csmu.edu.tw

$\triangle$ Hui-Ling Chiou

hlchiou@csmu.edu.tw

1 School of Medical Laboratory and Biotechnology, Chung Shan Medical University, 110, Section 1 Chien-Kuo N. Road, Taichung 402, Taiwan, ROC

2 Institute of Biochemistry and Biotechnology, Chung Shan Medical University, Taichung 402, Taiwan, ROC

3 Department of Pathology \& Laboratory Medicine, MacKay Memorial Hospital Hsinchu Branch, Hsinchu 690, Taiwan, ROC

4 Department of Biochemistry, Chung Shan Medical University, Taichung 402, Taiwan, ROC

5 Department of Medical Research, Chung Shan Medical University Hospital, Taichung 402, Taiwan, ROC

6 Department of Clinical Laboratory, Chung Shan Medical University, Taichung 402, Taiwan, ROC
Figure 5B and D, the wrong p-JNK and beta-actin panels in A549 and H1299 appear as the other panel. In Figure 5C, the wrong p-mTOR, mTOR and beta-actin panels appear as the other panels. All of the errors were caused during the manuscript preparation. The corrected figures do not affect the results in the published original article.

The corrected Figures are given below.

\section{New Figure 2D}
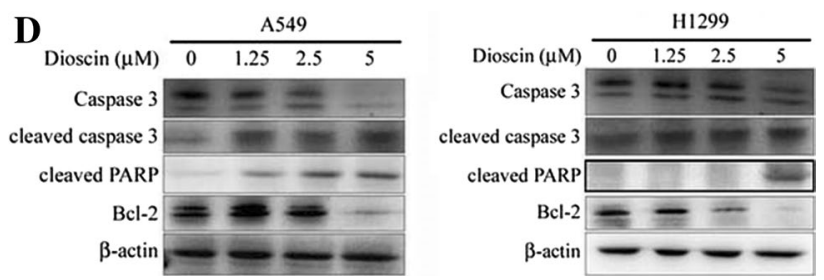

New Figure 3A

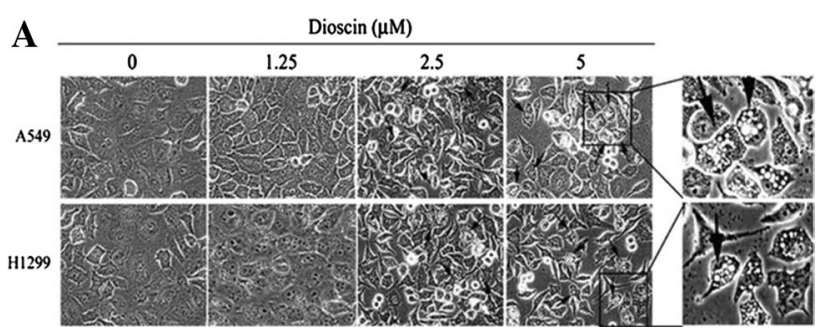




\section{New Figure 5ABCD}

A
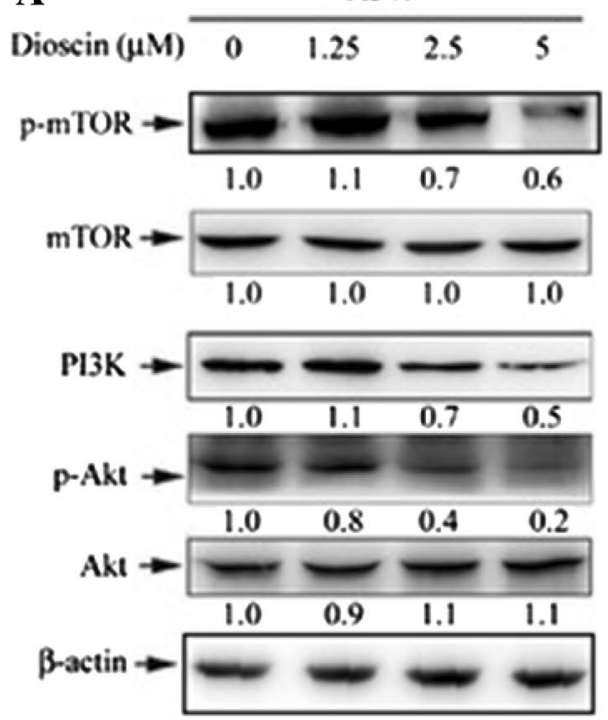

C
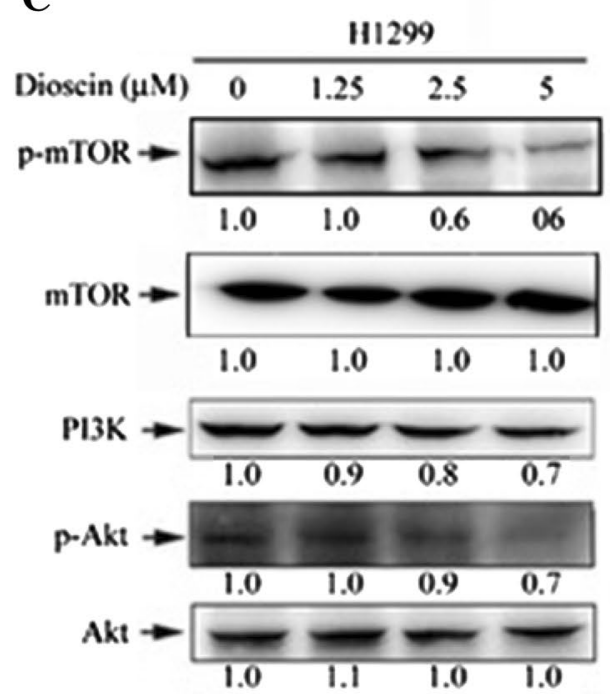

$\beta$-actin $\rightarrow-\begin{array}{llll}1.0 & 1.1 & 1.0 & 1.0 \\ & & & \end{array}$ \begin{tabular}{lllll} 
B & \multicolumn{4}{c}{ A549 } \\
\cline { 2 - 5 } Dioscin $(\mu \mathrm{M})$ & 0 & 1.25 & 2.5 & 5
\end{tabular}
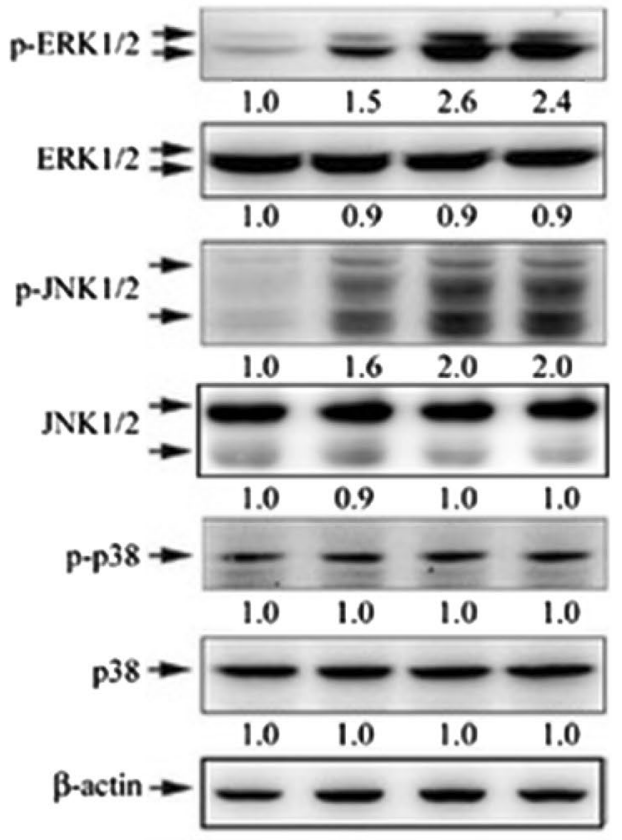

D
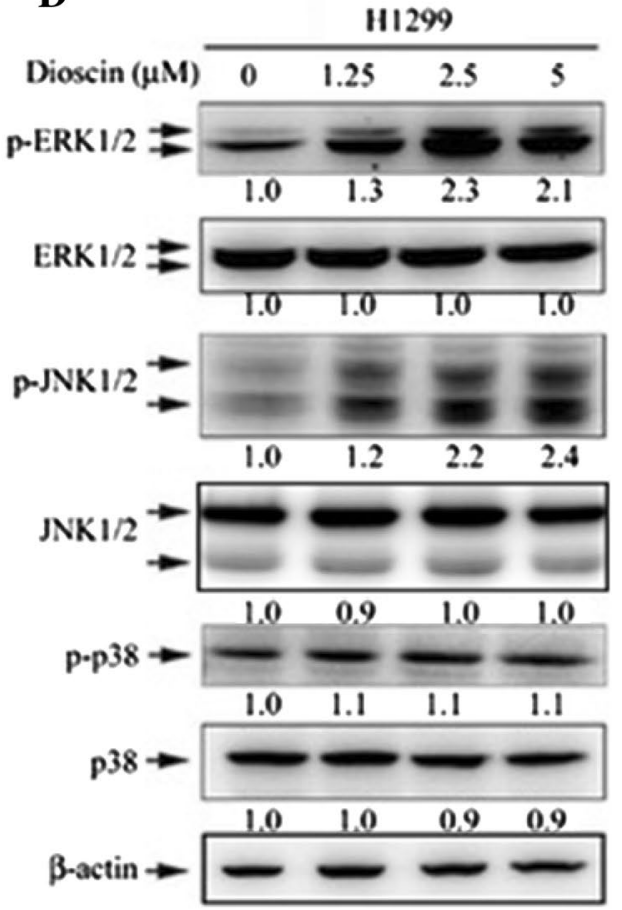